\title{
Dynamic modelling of complex systems under deep uncertainty using an exploratory multi-method approach
}

\author{
$\underline{\text { E.A. Moallemi }}^{\text {a }}$, S. EI Sawah ${ }^{\text {a }}$ and M.J. Ryan ${ }^{\text {a }}$ \\ ${ }^{a}$ Capability Systems Centre, School of Engineering and Information Technology, The University of New \\ South Wales, Canberra, Australia \\ Email: e.moallemi@unsw.edu.au
}

\begin{abstract}
The decision making of complex systems is challenging because of the presence of non-linearities and time delays in their structure and their behaviour. This decision making over the system lifetime is also challenged by the presence of deep uncertainty in the future behaviour of systems and in their surrounding environment. Traditional modelling approaches are inclined to consolidate all facts into a single ultimate model and to take a deterministic, optimal and predictive approach in decision making. However, they proved to be inadequate for coping with complexity and uncertainty challenges. We argue that an exploratory multi-method approach to modelling is needed for making effective and robust decisions for complex systems; the decisions which remain valid under a diverse range of future conditions. This paper illustrates the combined use of multimethod modelling and exploratory analysis in the support of complex systems decision making, with an application to asset acquisition and management and using the case of aircraft fleet as an illustrative example. First, a framework is introduced for the implementation of this multi-method exploratory approach in practice, and the model structure, developed for the case of aircraft fleet, is explained. We then discuss how the use of our new approach can improve the robustness of decisions in asset acquisition and management. An initial exploratory analysis is performed on the model under deep uncertainty conditions and with three design strategies: High Acquisition - Low Maintenance, Low Acquisition - High Maintenance, and Medium Acquisition - Medium Maintenance. The analysis of the results shows that investing on the maintenance capacity of an aircraft fleet could result in more average flying hours compared to more acquisition of new aircraft. However, this could cause two side-effects: a higher total (acquisition and maintenance) costs and a wider uncertainty in the future performance of the system (in terms of average flying hours and total costs).
\end{abstract}

Keywords: $\quad$ Robust decision making, exploratory modelling, system dynamics, multi-method modelling 
Moallemi et al., Dynamic modelling of complex systems under deep uncertainty using an exploratory multimethod approach

\section{INTRODUCTION}

Systems that operate in society, (such as food, energy, healthcare, and supply-chain systems) are influenced by human decisions to evolve towards the fulfilment of desired objectives, known as societal needs (de Haan et al., 2014). However, our understanding of these systems is limited, and their decision making is challenging. Human decisions often solve one issue at the expense of creating several other problems. The decisions may also work sufficiently at one time, but they mostly do not remain valid as new conditions unfold over time. These challenges are rooted in the complexity that lies in the systems' structure. These systems comprise a large number of elements within multiple social, technical and economic dimensions and linked to each other through chains of causal relations (Bunge, 1979; Moallemi et al., 2015). Another reason for the challenges is the complexity of the systems' behaviour. The systems generate non-linear patterns, side-effects and emergent characteristics, where the behaviour of a system cannot be explained by the behaviour of its individual elements (de Haan, 2006). These challenges are also due to deep uncertainties, i.e. conditions where the future states of the system structure are unknown or decision makers cannot agree on them (Maier et al., 2016).

Traditional approaches to decision making in complex systems use a 'consolidative approach' where all known facts are integrated into a single unifying model (Bankes, 1993). The consolidative approach results in an optimised decision based on an ultimate model. It tends to maximise the understanding of system behaviour by modelling a high-degree of detail using the best suitable modelling technique. Also, traditional approaches rely on a 'predictive approach' to cope with the uncertainty of the systems by drawing a best-guess estimate or a limited number of scenarios for the future. While these approaches seem to be valid from a purely engineering perspective, they often end up with complicated models, an increased cost of modelling, and unreliable decision advice in real-world problems, which would eventually cause scepticism to decision support tools and frameworks.

New approaches to decision making have emerged that suggest new ways of thinking about future decision making, enabling exploratory thinking, challenging decision maker's well-established assumptions, and investigating the unfolding of many plausible futures. We argue that a multi-method approach to modelling with this new exploratory approach for the investigation of results can deal with the decision making of complex systems more efficiently and robustly than traditional approaches. Using multiple modelling approaches enable us to cover the limitations of individual techniques in coping with the complexity at different levels of aggregation: actors' behaviours in a lower level of aggregation and the causal interactions of their accumulated behaviours in a higher level of aggregation. On the other hand, exploratory analysis can address the limitation of traditional scenarios where an accurate prediction of a single or few future conditions is impossible. It enables us to enhance the robustness of decisions by exploring an ensemble of system's behaviours over a large number of assumptions about the future. Accordingly, this paper illustrates the use of the exploratory multi-method approach in complex systems decision making under deep uncertainty. It shows the application of this approach in asset acquisition and management systems and with the management of aircraft fleet as an illustrative example. A combined system dynamics and discrete event approach within an object-oriented framework is chosen for dynamic modelling as they are methods capable of capturing complexities at low and high levels of aggregation (Sadsad et al., 2014; Scholl, 2001; Shafiei et al., 2012). The contribution of our new approach in this application is to enable decision makers to examine the future performance of the aircraft fleet in response to various design strategies (such as the mix of number of new acquisitions and capacity of maintenance lines) and under operational deep uncertainties (such as risk of loss during operation and variation of time between maintenances).

In the remainder of this paper, Section 2 explains the methods used for modelling and analysis. Section 3 presents the model structure. Section 4 shows the results of computational experiments with the model and then discusses the results with an exploratory framework. Section 5 concludes the paper.

\section{METHODS}

The exploratory multi-method approach is a combined use of system dynamics and discrete event approach for modelling and the use of exploratory analysis for the investigation of the results. In implementing our suggested approach, the dynamics of systems' interactions are initially conceptualised qualitatively by the engagement of stakeholders through expert workshops. This conceptualisation is formalised with modelling methods and implemented in a simulation platform. We combined the strengths of multiple methods in an object-oriented framework in AnyLogic 7.3. The object-oriented framework enables a rapid model building with a modular architecture and easily replicable to other contexts. It also facilitates the interactions of the modelling approaches at different levels of aggregation. On one hand, the discrete event approach allows for investigating the dynamics of the system caused by events and the interactions between the system entities. On the other hand, the system dynamics approach captures the feedback loops, created between the accumulation of system 
Moallemi et al., Dynamic modelling of complex systems under deep uncertainty using an exploratory multimethod approach

behaviour, such as the interactions between the average flying hours of aircraft and their impacts on the ageing of the fleet (Sterman, 2000). The main challenge of system dynamics models is in their limitation to incorporate heterogeneity and to develop dynamic model structures (Moallemi et al., 2015). The main challenge for the discrete event is the limited treatment of systems delays and feedback loops. For comparative studies between SD and DES, readers are referred to (Chahal \& Eldabi, 2008; Tako \& Robinson, 2010). The model's objective is to provide users with a library of modules that allow users to design a capability and constituent resources (e.g. personnel), and examine the performance of a capability, under a range of management options and scenarios over the capability lifecycle. The model has an object-oriented architecture, which include three types of software components:

(1) Decision-making component represent library of decision rules that can be used to control the processes that influence the capability through its lifecycle (i.e. controllers).

(2) Physical asset component is a blueprint of the most likely physical pathways a particular resource type can take (i.e. material, personal, personnel). The module is includes stock-flow representation of the processes that influence the availability for each basic resource type (See next section for details).

(3) Performance measures component is a library of functions that represent relevant performance indicators (e.g. total flying hours, and number of aircraft available for operation).

Detailed description of the model's architecture can be found in (ElSawah \& Ryan, 2016). When the model is developed, exploratory analysis is used to investigate the future performance of the systems in response to different design decisions and under uncertainty. Exploratory analysis is a new way of thinking about the future of systems under deep uncertainty; a situation where we do not or cannot agree about the future values, and we cannot represent them with a probability distribution (Lempert et al., 2003). Exploratory analysis studies the future behaviour of systems through 'exploration' and by answering 'what could happen?' instead of through 'prediction' and 'what will happen?' (Maier et al., 2016).To conduct exploratory analysis, the key uncertainties of the system and their possible ranges of variation are identified. Monte Carlo experiments are performed using the model and for the identified uncertainties. Exploratory analysis then uses statistical and data-mining techniques to interpret the extensive experiments and to suggest plural and conditional decision advice (J. H. Kwakkel \& Pruyt, 2013).

\section{MODEL DESCRIPTION}

In this paper, we use aircraft fleet management as an illustrative example. The model is designed in eight modules (see Figure 1): Design Variant, Acquisition, Flying Hours Allocation, Deep Maintenance (DM), Operational Maintenance (OM), Capability Assurance Program (CAP), Ageing and Retirement, and Loss. Each component is explained briefly as follows.

- Design Variant: Each variant represents different design variables specified by decision makers-for example, aircraft type, fleet size, acquisition and retirement plans, maintenance schedules, and operation conditions. Design Variant provides the required inputs, from decision makers, for all other modules.

- Acquisition: This module analyses the new purchases and the cost associated to them. The number of acquired aircraft is entered initially by decision makers as a variable of Design Variant. Acquisition adds additional aircraft to the fleet at the planned time during simulation. It then analyses the impact of new additions on the system performance.

- Flying Hours Allocation: This module determines the flying hours assigned to each aircraft based a required rate of effort that decision makers specify as a variable of Design Variant. It also uses a simple stock and flow structure to model the accumulation of the flying hours of aircraft over time to calculate the total flying hours.

- Deep Maintenance (DM) and Operational Maintenance (OM): DM and OM are condition-based activities with a same structure. This module models the time that an aircraft is due for maintenance (scheduling), the waiting queues for maintenance, and the maintenance process. It also analyses the cost associated to maintenance activities. The module uses inputs from Design Variants - for example, time between maintenances, the time spent by an aircraft in maintenance, and the available maintenance capacity.

- Capability Assurance Program (CAP): CAP is an upgrade program which assures the effectiveness of aircraft throughout its life. CAP affects the aircraft's total number of flying hours by bringing the aircraft's state back to the new condition or to a particular state determined by the decision maker. This module models the CAP process (such as the number of aircraft in CAP and the waiting queues for CAP) to examine the effects of various CAP arrangements on fleet performance.

- Ageing and Retirement: This module uses a system dynamics stock and flow structure to model the ageing of fleets according to an exponential decay function over the expected lifetime of the fleet. It differentiates 
Moallemi et al., Dynamic modelling of complex systems under deep uncertainty using an exploratory multimethod approach

between new, middle age, and old conditions of aircraft. The ageing process is influenced by the maximum flying hours and the required rate of effort, set by decision makers as a variable of Design Variant. Ageing also models the impact of aircraft' retirement considering the time when the total flying hours exceed the maximum flying hours.

- Loss: This module analyses the impact of the risk of loss of aircraft, during operation, on the system performance.

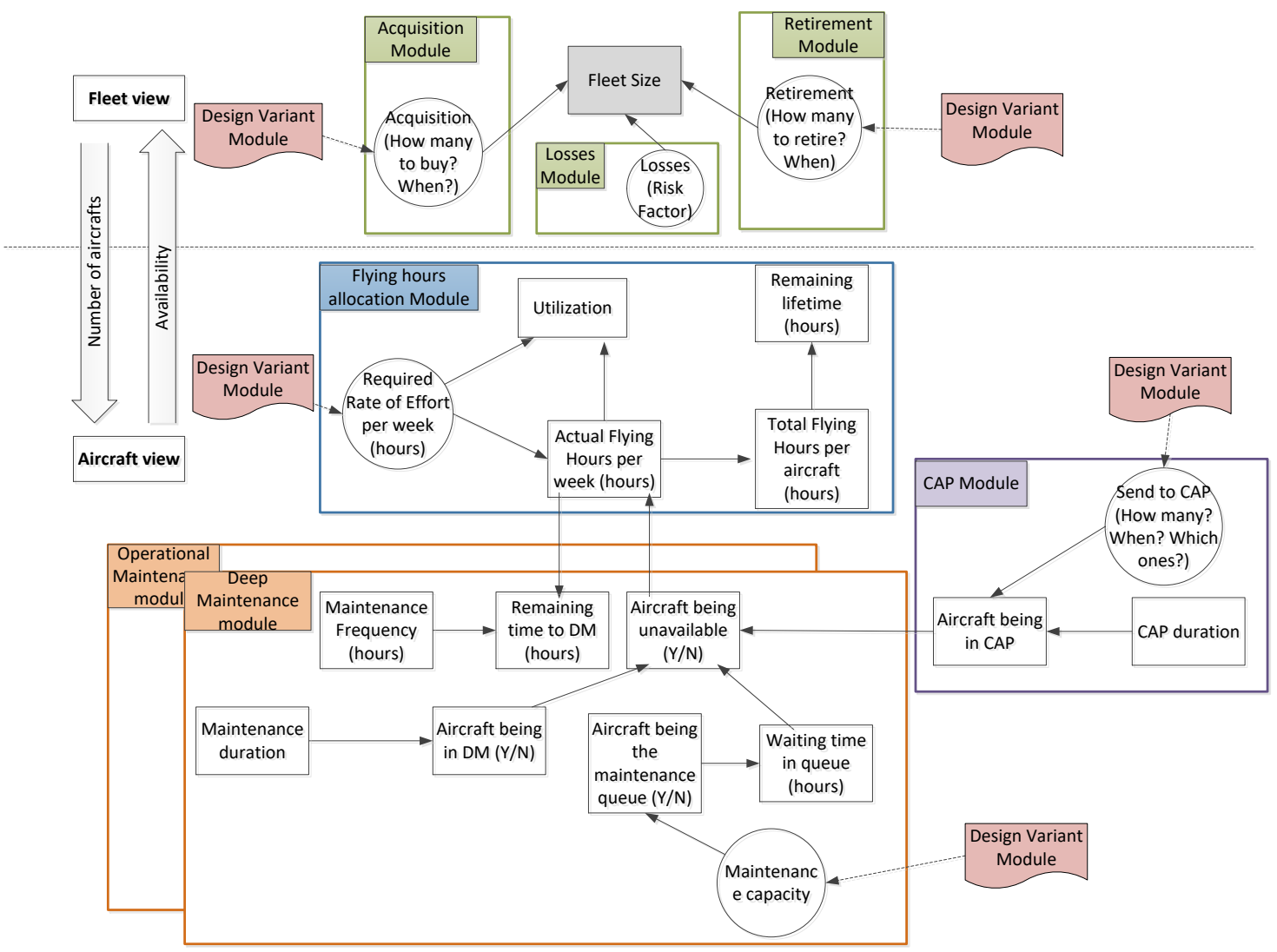

Figure 1. The high-level model architecture

\section{THE RESULTS OF EXPLORATORY ANALYSIS}

The future performance of the system (the aircraft fleet, in this case) varies depending on the chosen design strategies, i.e. the combinations of different values for new acquisition and maintenance capacity. The question of interest is how different strategies affect the performance of the system. The desire of decision makers to reach a deterministic picture from the impacts of different strategies is not possible under deep uncertainty (Stirling, 2010). Simplifying uncertainties for the sake of having definitive conclusions would result in apparently appealing but practically erroneous advice. We adopted exploratory analysis to avoid this issue and to present plural and conditional advice to assist decision makers in choosing between design strategies.

We used the model, developed in Section 3, to explore the impact of three maintenance and acquisition strategies (see Table 1) on the performance of the system: High Acquisition - Low Maintenance; Medium Acquisition - Medium Maintenance; and Low Acquisition - High Maintenance. The performance is expressed in terms of the total cost of new acquisition and maintenance, the number of aircraft in service, and the size of waiting queues for deep and operational maintenances. A dataset was compiled from different sources for model simulations. Seven hundred Monte Carlo experiments were performed with AnyLogic for the specified range of uncertainties (see Table 2) over a model time-horizon of 800 weeks. The experiments capture a wide space of conditions within which the system can operate. We then used a Python package, known as the EMA Workbench (J. Kwakkel, 2016), for the exploratory analysis of the experiments. Figure 2(a) and Figure 2(b) represent the average flying hours of the aircraft in a fleet and their total maintenance and acquisition costs under different strategies. We used histograms with kernel density estimates (KDE) to represent the diversity of the system performance in each variable. 
Moallemi et al., Dynamic modelling of complex systems under deep uncertainty using an exploratory multimethod approach

Figure 3 shows the distribution of the number of aircraft in-service and the size of waiting queues for deep and operational maintenance, for all time, across experiments and for each strategy. We represented the state of these variables in each single experiment with a KDE. What we are interested in Figure 3 is the peak (i.e. the highest probability of the number of aircraft or the size of queues in lifetime) and how the experiments are populated around each the peaks (i.e. the highest likelihood for the number of aircraft or the size of queues across all experiments).

\begin{tabular}{|c|c|c|c|}
\hline Parameters & $\begin{array}{l}\text { Strategy I: High } \\
\text { Acquisition - Low } \\
\text { Maintenance }\end{array}$ & $\begin{array}{l}\text { Strategy II: Medium } \\
\text { Acquisition - Medium } \\
\text { Maintenance }\end{array}$ & $\begin{array}{l}\text { Strategy III: Low } \\
\text { Acquisition - High } \\
\text { Maintenance }\end{array}$ \\
\hline Number of new acquisition & 6 & 4 & 2 \\
\hline CAP capacity & 0 & 1 & 3 \\
\hline OM capacity & 1 & 2 & 3 \\
\hline DM capacity & 1 & 2 & 3 \\
\hline
\end{tabular}

Table 2. List of uncertain parameters and their ranges of variation (+-50\%)

\begin{tabular}{ll}
\hline Uncertain parameter & Range \\
\hline The risk that that an aircraft is lost during operation & $0.00065-0.00195(-)$ \\
The life time of aircraft & $93600-280800$ (hour) \\
A minimum and maximum for total required flying hours with a uniform distribution & $30-91 ; 42-126(-)$ \\
Expected time spent by an aircraft in CAP & $12-36$ (week) \\
The time between CAP events & $10-30$ (week) \\
Expected time spent by an aircraft in DM & $8-12$ (week) \\
The time (flying hours) between DM events & $500-1500$ (hour) \\
Expected time spent by an aircraft in OM & $2-5$ (week) \\
The time between OM events & $125-375$ (hour) \\
Cost of OM & $100,000-2,000,000(\$)$ \\
\hline
\end{tabular}

We assumed that the decision makers in our example have a number of objectives: increasing the average flying hours of the fleet while minimising the costs associated with it; maintaining a high number of aircraft in-service while avoiding long waiting queues in maintenance lines. Based on the results of exploratory analysis in the pre-defined strategies (see Figure 2 and 3), none of strategies can satisfy all objectives at the same time. The performance of three strategies can be analysed by making a trade-off among the fulfilment of multiple objectives. As an example, we analysed this performance trade-off in Strategy III (Low Acquisition High Maintenance) as follows:

- First: investing in maintenance capacity could be a more effective strategy for improving the availability of in-service aircraft and the average flying hours compared to buying new aircraft. This could also reduce the waiting queues for maintenances. The total aircraft in-service (see

- Figure 3(a)) in Strategy III (with a high maintenance capacity) is distributed around three with a variation to 10 aircraft while the distribution of aircraft in-service in Strategy I (with a high acquisition rate) has a mean of one and can vary to a maximum of six aircraft. Also, the average flying hours in Strategy III (see Figure 2(a)) has a distribution populated around a higher value (mean: 8000 hours) compared to Strategy I (mean: 2000 hours). This behaviour can be explained by the fact that the acquisition of new aircraft increases the pressure on the maintenance lines and creates a buffer of long waiting queues. The longer queues in a strategy with high numbers of acquisitions are evident in 3(b) and 3(c) where there is a local maximum of 10 and 12 aircraft in deep and operational maintenance queues.

- Second: a design strategy with emphasis on maintenance capacity could end up with higher total costs compared to a strategy with a larger number of new acquisitions. This is reflected in Figure where the mean of total costs in Strategy III is around $\$ 900$ billion compared to about $\$ 500$ billion total costs in Strategy I. The extent to which this increase of costs could work against the desirability of Strategy III depends on the sensitivity of decision makers to different objectives in their decision making process.

- Third: a design strategy with larger maintenance capacity could result in a wider uncertainty in the future performance of the system, in terms of average flying hours and total cost. The implication of this is that increasing the maintenance capacity is not a preferred strategy if decision makers expect more deterministic advice. Figure 2(a) shows that Strategy III has a higher mean of average flying hours compared to the other strategies, but it is subject to wider uncertainty, a range from 2000 to 30000 hours (where this varies between around 1000 to 10000 in Strategy I). 
Moallemi et al., Dynamic modelling of complex systems under deep uncertainty using an exploratory multimethod approach

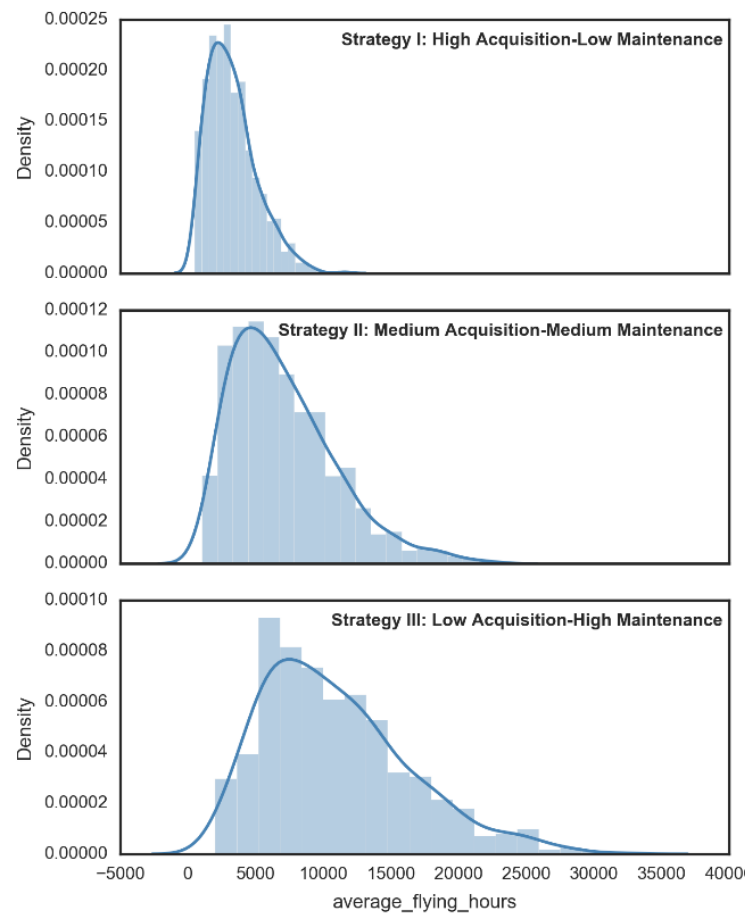

Figure 2(a). Histogram and KDE for average flying hours of the aircraft (hours)

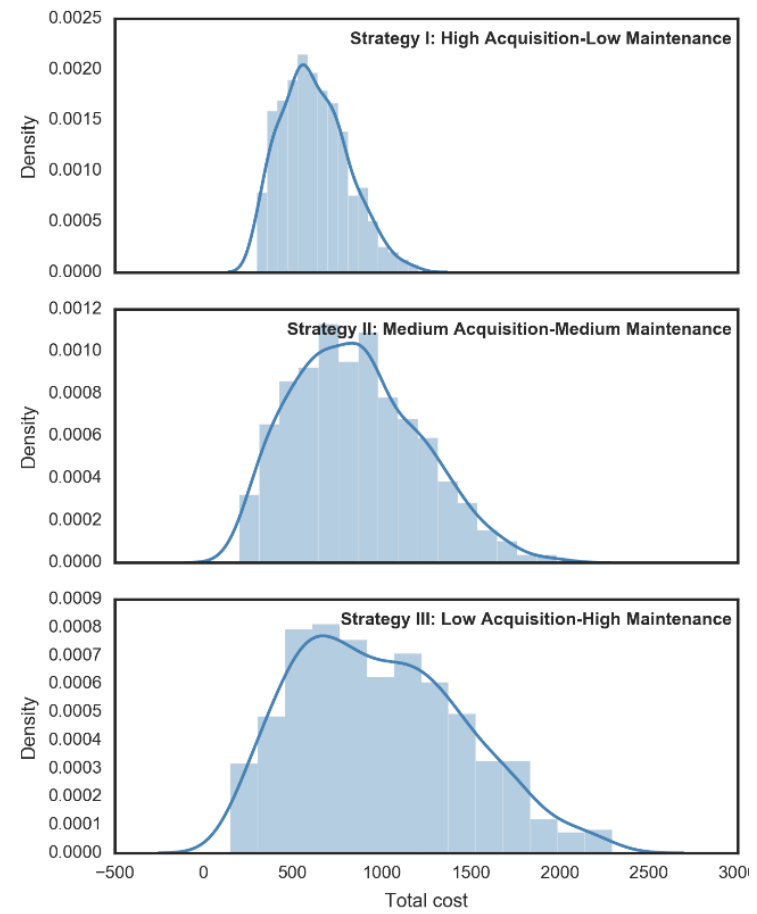

Figure 2(b). Histogram and KDE for total acquisition and maintenance cost (\$billion)

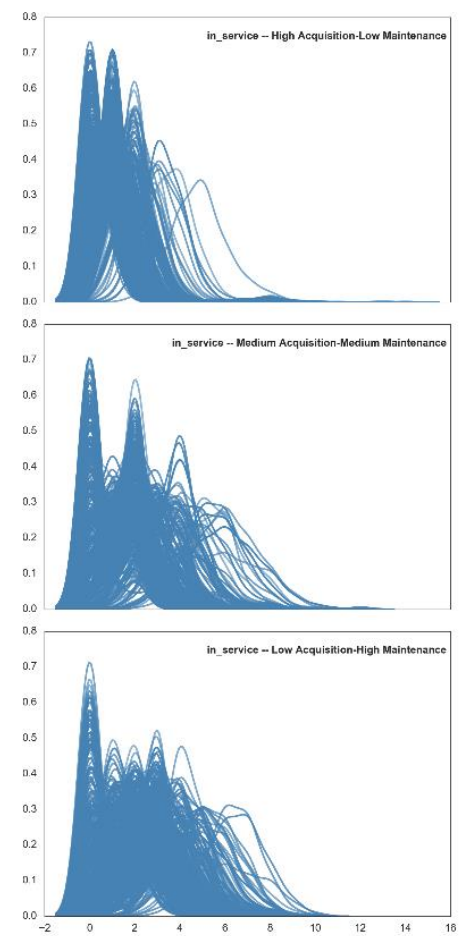

(a)
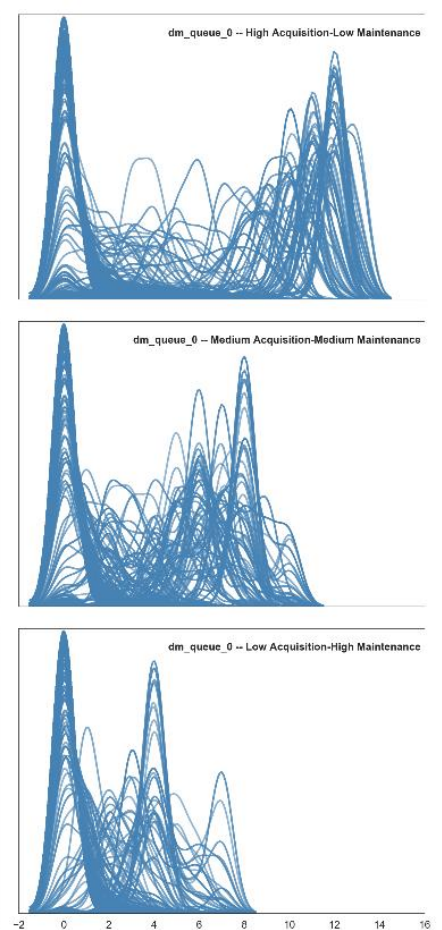

(b)
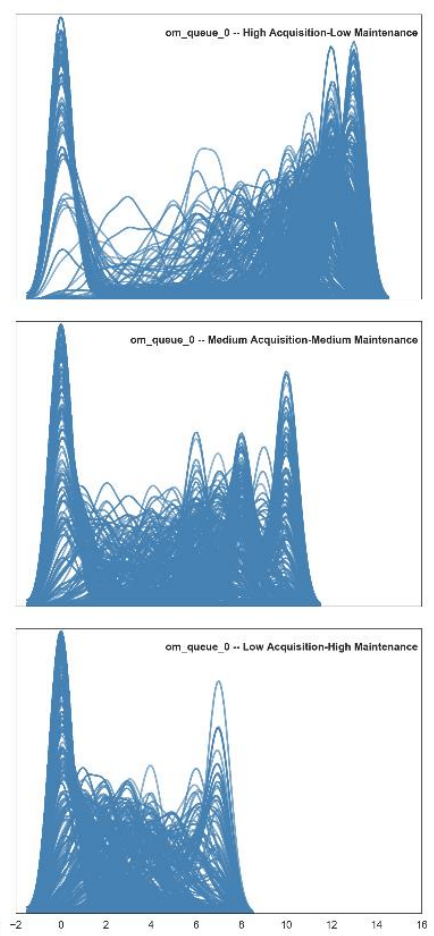

(c)

Figure 3. Kernel density estimates for the number (a) of aircraft in service, (b) in the deep maintenance queue, and (c) in the operational maintenance queue, in a period of 800 weeks

\section{CONCLUSIONS}

The decision making of complex systems is challenging because of their complexity-driven characteristics and the uncertainties in their surrounding environment. We discussed that a traditional consolidative system 
Moallemi et al., Dynamic modelling of complex systems under deep uncertainty using an exploratory multimethod approach

modelling with a predictive/deterministic approach for the future-oriented analysis of results is complicated, costly and erroneous. We argued that: (1) a multi-method approach to modelling can improve the limitation of each separated modelling techniques in explaining system complexity, and (2) an exploratory analysis of modelling results can cope with deep uncertainty and propose plural decision advice that work under diverse plausible futures. To explain our proposed exploratory multi-method modelling approach, a combined discreet event and system dynamics model was developed for asset acquisition and management systems with an illustrative example in aircraft fleets. We performed an exploratory analysis for system's uncertainties and concluded that a design strategy based on increasing the size of maintenance capacity could lead to higher average flying hours in a fleet but also higher total costs compared to a strategy with the larger number of new acquisitions. A limitation of the current analysis is in a way that we proposed decision advice by comparing design strategies with three sets of pre-defined values for the number of new acquisition and size of maintenance capacity. Decision makers, in reality, do not have presumed strategies in advance. Instead, they expect the strategies to be suggested by decision support tools. A future research is to show how our exploratory multi-method approach can suggest robust design strategies by making a trade-off between the number of new acquisitions and the size of maintenance capacity, in the process of exploratory analysis.

\section{REFERENCES}

Bankes, S. (1993). Exploratory modeling for policy analysis. Operations Research, 41(3), 435-449.

Bunge, M. A. (1979). Causality and Modern Science (3rd ed.): Dover Publications.

Chahal, K., \& Eldabi, T. (2008). Applicability of hybrid simulation to different modes of governance in UK healthcare. Paper presented at the Simulation Conference, 2008. WSC 2008. Winter.

de Haan, F. J. (2006). How emergence arises. Ecological Complexity, 3(4), 293-301. doi: http://dx.doi.org/10.1016/j.ecocom.2007.02.003

de Haan, F. J., Ferguson, B. C., Adamowicz, R. C., Johnstone, P., Brown, R. R., \& Wong, T. H. F. (2014). The needs of society: A new understanding of transitions, sustainability and liveability. Technological Forecasting and Social Change, 85, 121-132. doi: http://dx.doi.org/10.1016/j.techfore.2013.09.005

ElSawah, S., \& Ryan, M. (2016). A modular and approach to dynamic modelling approach for capability planning. Paper presented at the Australasian Simulation Congress SimTect 2016, Melbourne, 26 29 September.

Kwakkel, J. (2016). Exploratory Modelling and Analysis (EMA) Workbench: TU Delft.

Kwakkel, J. H., \& Pruyt, E. (2013). Exploratory Modeling and Analysis, an approach for model-based foresight under deep uncertainty. Technological Forecasting and Social Change, 80(3), 419-431. doi: http://dx.doi.org/10.1016/j.techfore.2012.10.005

Lempert, R. J., Popper, S. W., \& Bankes, S. C. (2003). Shaping the next one hundred years: new methods for quantitative, long-term policy analysis: Rand Corporation.

Maier, H. R., Guillaume, J. H. A., van Delden, H., Riddell, G. A., Haasnoot, M., \& Kwakkel, J. H. (2016). An uncertain future, deep uncertainty, scenarios, robustness and adaptation: How do they fit together? Environmental Modelling \& Software, 81, 154-164. doi: http://dx.doi.org/10.1016/j.envsoft.2016.03.014

Moallemi, E. A., de Haan, F., George, B., Webb, J., \& Aye, L. (2015). Dynamic modelling of energy transitions using a coupled modelling-narrative approach. Paper presented at the The 21 st International Congress on Modelling and Simulation (MODSIM2015), November 29 - December 4 2015, Gold Coast, Australia.

Sadsad, R., McDonnell, G., Viana, J., Desai, M., Harper, P., \& Brailsford, S. (2014). Hybrid modelling case studies.

Scholl, H. J. (2001, 3-6 Jan. 2001). Agent-based and system dynamics modeling: a call for cross study and joint research. Paper presented at the System Sciences, 2001. Proceedings of the 34th Annual Hawaii International Conference on.

Shafiei, E., Stefansson, H., Asgeirsson, E. I., Davidsdottir, B., \& Raberto, M. (2012). Integrated Agent-based and System Dynamics Modelling for Simulation of Sustainable Mobility. Transport Reviews, 33(1), 44-70. doi: 10.1080/01441647.2012.745632

Sterman, J. (2000). Business dynamics: systems thinking and modeling for a complex world. USA: IrwinMcGraw-Hill.

Stirling, A. (2010). Keep it complex. Nature, 468(7327), 1029-1031.

Tako, A. A., \& Robinson, S. (2010). Model development in discrete-event simulation and system dynamics: An empirical study of expert modellers. European Journal of Operational Research, 207(2), 784-794. 